\title{
Spatio-Temporal Patterns in the Depth EEG During the Epileptic Seizure*
}

\author{
Jung Ae Kim ${ }^{1}$, Sunyoung $\mathrm{Cho}^{2}$, Sang Kun Lee ${ }^{3}$, \\ Hyunwoo $\mathrm{Nam}^{3}$, and Seung Kee $\mathrm{Han}^{1,2}$ \\ ${ }^{1}$ Department of Physics, Chungbuk National University, \\ Cheongju, Korea, \\ ${ }^{2}$ Basic Science Research Institute, Chungbuk National University, \\ Cheongju, Korea, \\ ${ }^{3}$ Departments of Neurology, College of Medicine, Seoul National University, \\ Seoul, Korea, \\ sycho@chungbuk.ac.kr
}

\begin{abstract}
We analyzed the spatio-temporal patterns of the depth EEG recorded from a patient with lateral temporal-lobe epilepsy. Statistical analysis based on the Jensen-Shannon entropy (JS-E) as well as linear power spectral analyses were performed. The spatio-temporal patterns from JS-E and $\beta$ rhythm successfully detected the onset timing of the seizure and revealed the temporal topology of the epileptic focus. The robustness of these patterns was proved by inter-trial consistency. As the patterns are well matched with the clinical diagnosis, it could be used for the identification of onset time and focus region of epileptic seizure.
\end{abstract}

\section{Introduction}

The epilepsy is characterized by a disturbance on the electrochemical activity of the brain, producing an excessive and hypersynchronous activity of the neuron. Epileptic seizures reflect this sudden and recurrent malfunction of the brain areas. Partial (focal) seizures originate from a restricted region and remain to this region (epileptic focus), while generalized seizures involve almost the entire brain. Partial seizures generally originate at a cortical area; most of them begin focally at the temporal lobes.

Exact localization of the epileptic focus and its delineation from functionally relevant areas are required for successful surgical treatment and understanding the basic mechanism of the seizures. The primary tool for this purpose is the EEG due to high temporal resolution and close relationship to physiological and pathological function of the brain. Various spectral and nonlinear analyses of epileptic EEG have been used to detect the epileptic focus and to predict the seizure onset [1 4]. Investigators have extracted specific quantitative features from the EEG, which would be characteristics for the discharge patterns during seizure [5].

* This work was supported by Korean Research Foundation, KRF 2002-075-H0007 to S.Y. Cho, and a grant (M103KV010011-03K2201-01130) from Brain Research Center of the 21st Century Frontier Research Program funded by the Ministry of Science and Technology of Republic of Korea to S.K. Han. 
We analyzed the spatio-temporal patterns of the epileptic EEG recorded intracranial for the temporal topology and source localization of seizure initiation and propagation. Statistical analysis based on the Jensen-Shannon entropy (JS-E) and linear power spectral analyses were performed [6].

\section{Method}

\subsection{Intracranial Recording}

Our data was from a patients presenting lateral temporal epilepsy clinically diagnosed at Epilepsy Center in Medical collage of Seoul National University. Intracranial recording was required to confirm the exact site of the structures generating seizure onsets before surgical operation. The EEG recording were performed on 30 sites as depicted in Fig 1 and 2 (Telefactor Beehive telemonitoring system, sampling rate $200 \mathrm{~Hz}$, bandwidth filtering $0.1 \sim 70 \mathrm{~Hz}$ ). The data set analyzed in this study had duration of 3-5 min including $1 \mathrm{~min}$ before the seizure. The seizure onset was determined by electrographic criteria as localized, sustained rhythmic discharges (burst of rhythmic spikes, low-voltage fast rhythms, etc.) and associated with subsequent clinical seizure activity [7].

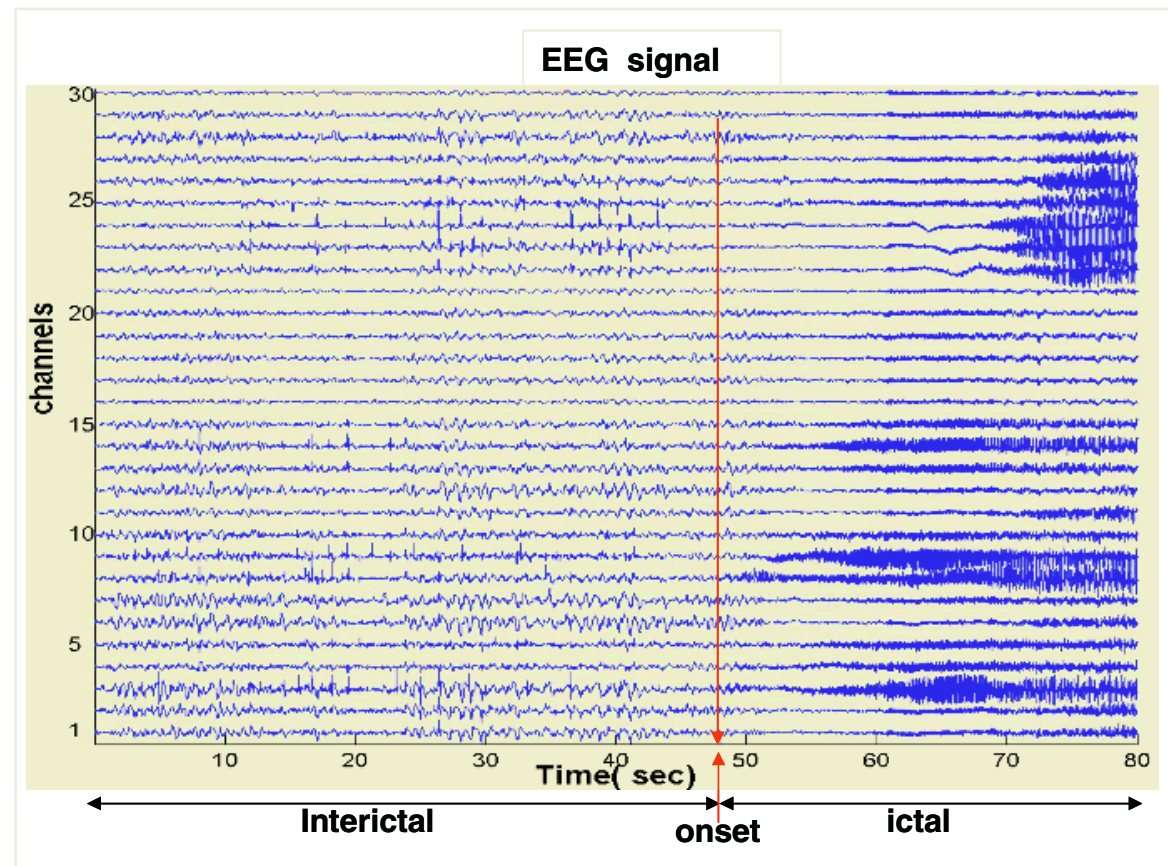

Fig. 1. Example of depth EEG raw data recorded from 30 sites on the temporal lobe. The red arrow (onset) points the EEG onset of seizure activity diagnosed clinically 


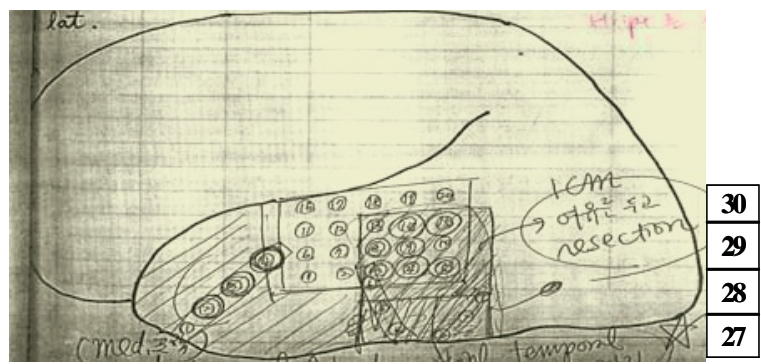

\begin{tabular}{|c|c|c|c|c|c|c|c|}
\hline 16 & 17 & 18 & 19 & 20 & & & \\
\hline 11 & 12 & 13 & 14 & 15 & & & \\
\hline 6 & 7 & 8 & 9 & 10 & & & \\
\hline 1 & 2 & 3 & 4 & 5 & & & \\
\hline & & 26 & 25 & 24 & 23 & 22 & 21 \\
\hline
\end{tabular}

Fig. 2. Schematic view of the recording sites on the temporal lobe. 21-26 sites were covered on the ventral area of inferial temporal region. The numbers of channels are shown in the left boxes

\subsection{Jensen-Shannon Entropy}

We applied the Jensen-Shannon entropy to analyze spatio-temporal patterns of the EEG time series data. Jensen-Shannon entropy (JS-E) is a statistical measurement that quantifies the difference between two (or more) probability distributions [8]. This value would be maximized when two distributions reveal the most prominent difference in the statistical characteristics.

In case of epileptic EEG, we can find the abrupt changing points of the time series data using JS-E. When total series $S$ is divided to two subset, $S_{1}$ and $S_{2}$ of lengths $1_{1}$ and $1_{2}$, JS-E is calculated from the entropies of total time range $(\mathrm{S})$ and two subsets $\left(\mathrm{S}_{1}\right.$ and $\left.\mathrm{S}_{2}\right)$ by equations below. With moving the dividing point by $1.5 \mathrm{sec}$, the distribution of JS-E was obtained. The EEG data in whole trial could be separated into sub periods using JS-E peak points (see Fig 6).

$$
z(t)=x(t+1)-x(t)
$$

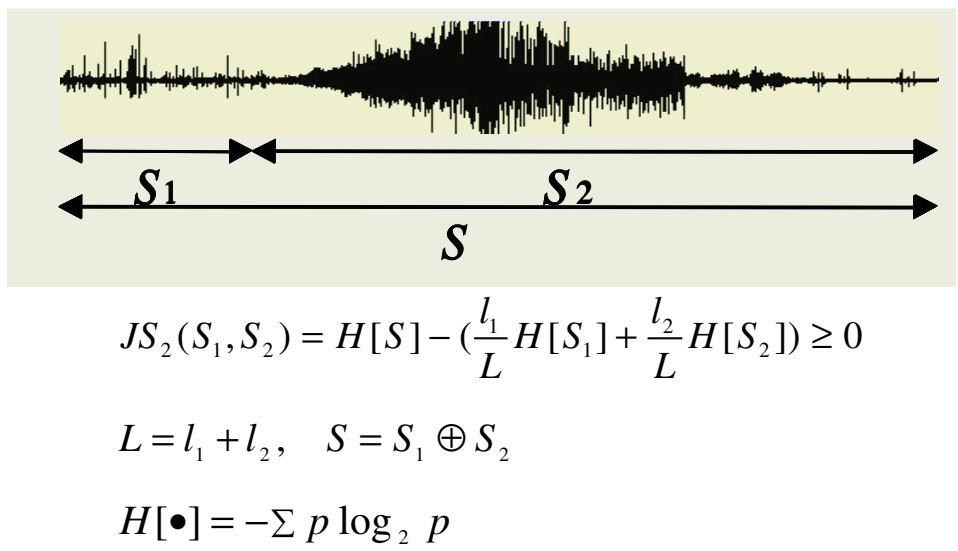

where $\mathrm{H}$ denotes Shannon entropy of the probability distribution $\{\mathrm{p}\}$. 


\section{Results}

For spectral analysis we considered the following frequency bands: theta $(\theta: 3 \sim 7 \mathrm{~Hz})$, alpha $(\alpha: 8 \sim 13 \mathrm{~Hz})$ and beta $(\beta: 20 \sim 30 \mathrm{~Hz})$. Fig. 3 displays the mean time course of powers (averaged in 30 channels) of total and three frequency bands with window size $10.24 \mathrm{sec}$ and $50 \%$ overlap. Fig. 4 displays temporal patterns of each band accumulated by sorted channels.

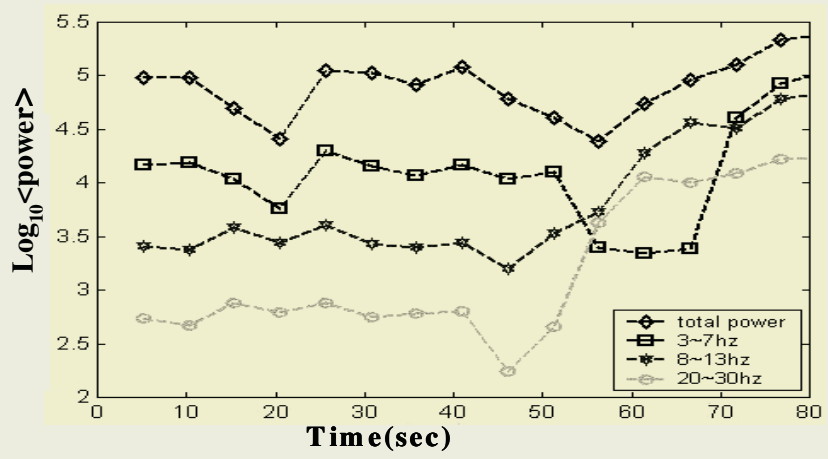

Fig. 3. The mean time course of powers of total and three frequency bands
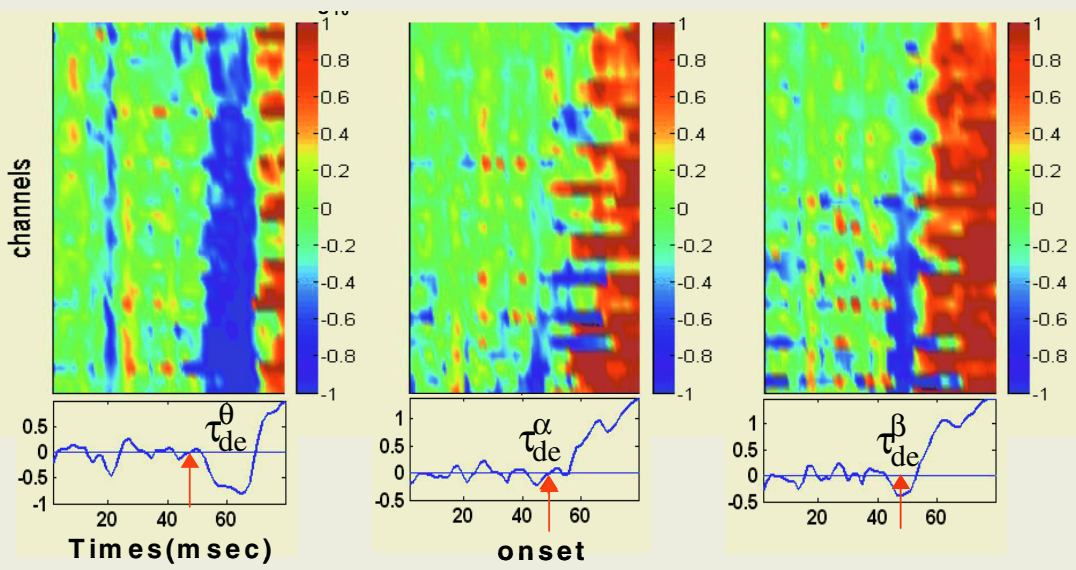

Fig. 4. Power spectral density accumulated by channels sorted by the amount of decrease after seizure onset. The averages on the channels were shown in the boxes below. The red arrow (onset) points the EEG onset of seizure diagnosed clinically

Near the onset time of seizure, $\beta$ rhythm started to decrease for a short time (about 5$10 \mathrm{sec}$ ) and followed by an increase as the seizure evolved. Fig 5 shows the change of power spectral topography of $\beta$ rhythm over time. These activities are well matched to 
the location of seizure focus defined clinically and reflect the progression of the seizure. Compare to $\beta$ rhythm, $\theta$ it started to decrease later and sustained longer.

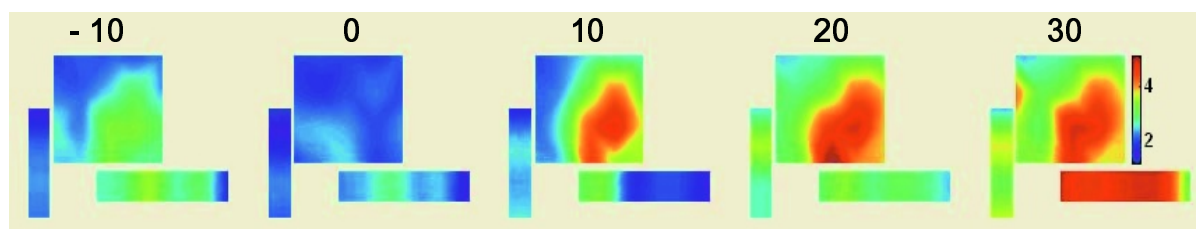

Fig. 5. Temporal evolution of power spectral topography of $\beta$ rhythm. The onset time is scaled zero and the window size is $10.24 \mathrm{sec}$ and $50 \%$ overlap

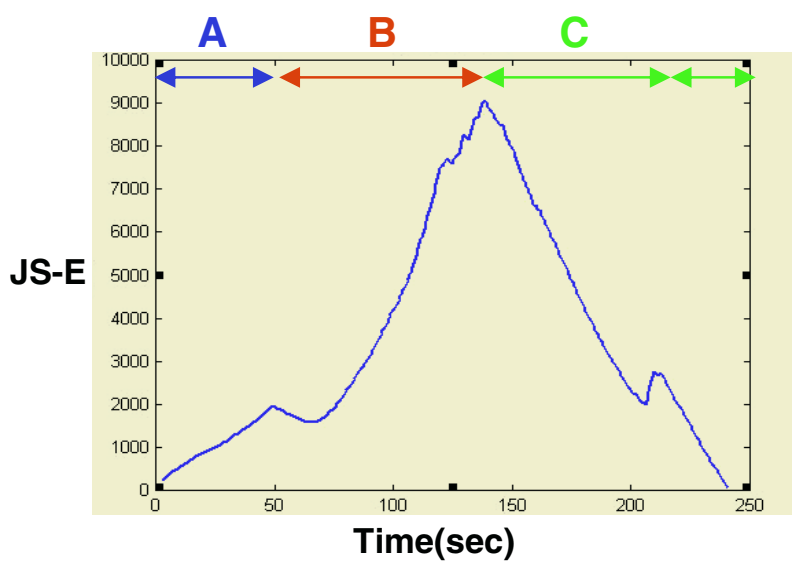

Fig. 6. JS-E mean distribution over time. Sub periods (A: inter-ictal, B: ictal, C: post-ictal period) could be identified using the peaks

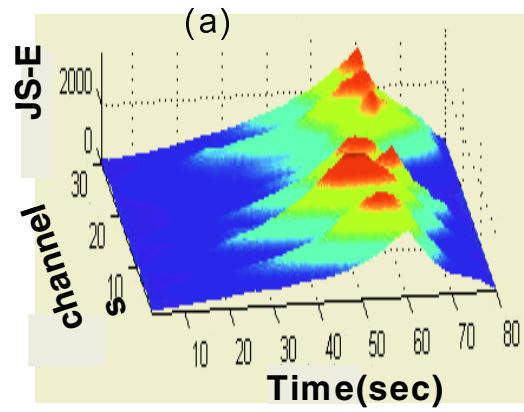

(b)

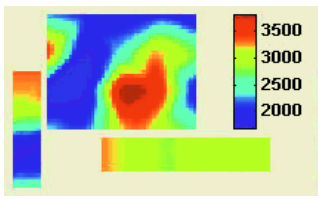

Fig. 7. JS-E distribution in each channel during early $80 \mathrm{sec}$ (a) and the peak entropy topography (b) 
In Fig 6, a whole trial EEG data were divided into sub periods using JS-E peak points. Those sub periods fitted the evolving stage of seizure (inter-ictal / ictal / postictal) very well. We analyzed further the data for the early period for the source localization of seizure initiation and propagation, that is 80 sec-duration around seizure onset. Fig 7(a) showed the distribution of JS-E across 30 channels over time, and Fig 7(b) depicted the peak entropy value on each recording site. It also matched well to the seizure focus.

We tried to prove the robustness of these patterns using inter-trial consistency. As shown in Fig 9, the JS-E peak entropy topography and $\beta$ power topography at the $200 \%$ power- increasing point were consistent across the trials.

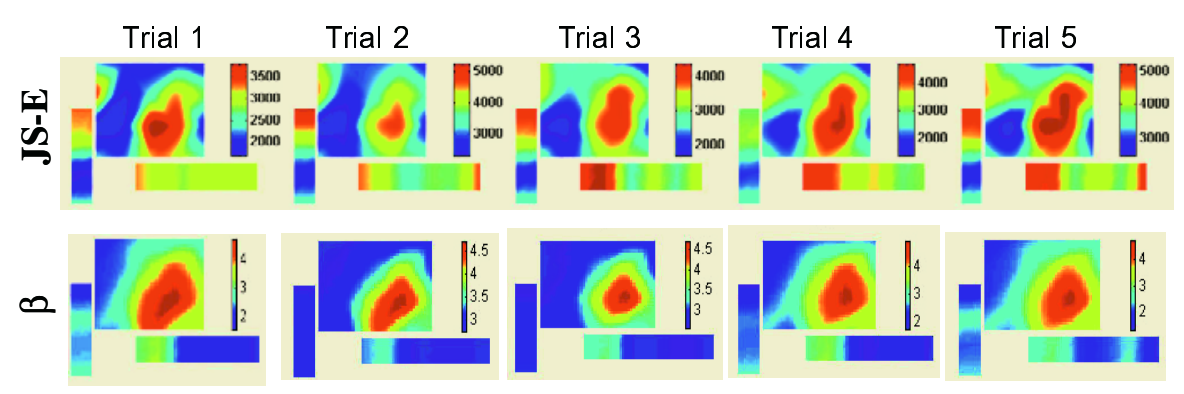

Fig. 8. JS-E peak entropy topography and $\beta$ power topography at the $200 \%$ power- increasing point in each trial, that were consistent across the trials as well as the exact localization of epileptic focus

\section{Discussion}

The spatio-temporal analysis using the Jensen-Shannon entropy successfully detected the onset timing of the seizure and revealed the topographic information of ongoing seizure. Besides the localization of epileptic focus it could be extended to the temporal profiles of seizure propagation.

In the spectral analysis, $\beta(20-30 \mathrm{~Hz})$ and $\theta$ rhythm $(3-7 \mathrm{~Hz})$ showed spatio-temporal patterns correlated with the ongoing seizure. $\alpha$ rhythm $(8-13 \mathrm{~Hz})$ showed similar patterns even much more moderately compare to other bands. They showed decrease near the onset time of seizure and followed by an increase as the seizure evolved. Especially $\beta$ rhythm started to decrease before the seizure onset and revealed spatiotemporal topography well matched to clinical diagnose of the epileptic focus and temporal propagation of seizure.

The robustness of these patterns was proved by inter-trial consistency, that is the spatio-temporal patterns from Jensen-Shannon entropy and $\beta$ rhythm were consistent across the trials. As the patterns are well matched with the clinical diagnosis, it could be used for the identification of onset time and focus region of epileptic seizure. 


\section{References}

1. Lerner, D. E. : Monitoring changing dynamics with correlation integrals:Case study of an epileptic seizure.Phys. D 97(1996) 563-576

2. Martinerie, J., Adam,C., Quyen, M. L. V. , Baulac,M., Clemenceau, ., Renault,B. and Varela, F.J.: Epileptic crisis can be anticipated by non-linear analysis, Nature Medicine vol.4 (1998) 1173-1176

3. Pereda, E., Rial, R., Gamundi, A., Gonzalez, J.: Assessment of changing interdependencies between human electroencephalograms using non-linear methods, Phys. D 148 (2001) 147.

4. Quyen, M. L. V., Martinerie, J, Baulac, M. Varela, F.: Anticipating epileptic seizures in real time by a non-linear analysis of similarity between EEG recordings, NeuroReport 10, (1999) 2149-2155

5. Mormann, F., Elger,C.E. and Lehenrtz, K.: Automatic detection of a preseizure state based on a decrease in synchronization in intracranial electroencephalogram recordings from epilepsy patients, Phys. Rev. E 67, (2003) 021912-1-10

6. Galvan, P. B., Grosse, I., Carpena, P., Oliver, J. L., Roldan, R.R. Stanley, H.E.: Finding borders between coding and noncoding DNA regions by an entropy segmentation method, Phys. Rev. Lett. 85(6), (2000) 1342-1345

7. Engel, C. E.; Seizure and epilepsy: contemporary neurology series. Philadelphia: FA Davis, (1989)

8. Grosse, I., Galvan, P. B., Carpena, P., Roldan, R.,R., Oliver, J., and Stanley, H., E.,: Analysis of symbolic sequences using the Jensen-Shannon divergence, Phys. Rev. E 65,(2002) 041905-1-16 